\title{
Incubation effort in relation to male attractiveness in zebra finches Taeniopygia guttata
}

\author{
Helen E. Gorman, Kathryn E. Arnold and Ruedi G. Nager
}

Gorman, H. E., Arnold, K. E. and Nager R. G. 2005. Incubation effort in relation to male attractiveness in zebra finches Taeniopygia guttata. - J. Avian Biol. 36: 413-420.

\begin{abstract}
The division of labour in parental care between the two sexes varies between and within species. In birds, parents have been shown to invest more into egg production and nestling care when paired with an attractive rather than an unattractive mate, as predicted by the differential allocation hypothesis. Here we investigate variation in the female's and male's share of incubation behaviour, a vital, and costly, period of parental care during which the embryo is vulnerable to perturbations in developmental conditions. We manipulated the attractiveness of male zebra finches Taeniopygia guttata, using red or green leg-rings. To simulate their natural social environment we allowed them to breed in outdoor aviaries. All males within an aviary were given the same coloured ring to avoid ring-colour related assortative mating. Males within a colony, however, were still expected to show some variation in attractiveness with the earliest laying females possibly pairing with the most attractive males. Indeed we found that both factors played a role in explaining female incubation effort. Among females mated to red ringed males, earlier laying females contributed significantly more to incubation than late laying females, but no such pattern was found in females mated to green ringed males. Overall, there were no differences in the level of incubation provided by both parents between treatment groups, suggesting some compensation within the pair. Hatching success was correlated with a pair's total incubation effort. These results suggest that variation in the division of parental care between the sexes is in agreement with both increased effort of females mated with attractive males, and females compensating for the reduced effort of attractive males seeking further mating opportunities. These two factors can act at the same time in natural populations and both should be considered when explaining variation in division of labour between the sexes.
\end{abstract}

H. E. Gorman, K. E. Arnold and R. G. Nager (correspondence), Division of Environmental \& Evolutionary Biology, Institute of Biomedical and Life Sciences, Graham Kerr Building, University of Glasgow, Glasgow, G12 8QQ, U.K. E-mail: r.nager@bio.gla.ac.uk

Parental care is a fundamental determinant of fitness. The resources provided by parents will have far-reaching consequences for offspring. By the same token, caring is expensive for parents and will influence their future survival and reproduction. Thus, parents need to strategically allocate resources between offspring and breeding attempts to maximise net fitness gains. The division of parental effort between male and female is variable among and within species, and the source of this variation is not well understood (Clutton-Brock 1991).
There are many potential causes of variation in parental care and several studies have focused on how the sexual attractiveness of an individual, usually the male, influences the parental investment decisions of its mate (Burley 1986, 1988a, Sheldon 2000). Attractiveness can be a signal of male quality, implying that the male will provide superior genes to his offspring (Zahavi 1975, Hamilton and Zuk 1982, Andersson 1986, Jennions and Petrie 2000), or alternatively that he is able to supply high-quality parental care (Hoelzer 1989) and/or resources needed for optimal offspring development 
(Norris 1990, Keyser and Hill 2000, Siefferman and Hill 2003; but Smiseth et al. 2001). Consequently, it will be advantageous for a female to mate with an attractive male. The differential allocation hypothesis suggests that selection should favour individuals that allocate more resources to their offspring when paired with an attractive mate (Burley 1986, 1988a, Sheldon 2000). Evidence for the differential allocation hypothesis has been found in many taxa, including insects (Simmons 1987), amphibians (Reyer et al. 1999), mammals (Drickamer et al. 2000) and fish (Kolm 2001), but the majority of studies have been carried out on birds (Sheldon 2000 for a review, and references below). In birds, increased mate attractiveness has been shown to affect female copulation frequency (Torres and Velando 2003), allocation of resources into egg formation (Petrie and Williams 1993, Zann 1994, Balzer and Williams 1998, Gil et al. 1999, Cunningham and Russell 2000, Saino et al. 2002a, b; but Norris 1990, Swaddle and Cuthill 1994) and post-hatching parental care (Burley 1988a, de Lope and Møller 1993, Swaddle 1996; but Sanz 2001, Rohde et al. 1999). No studies, however, have specifically considered incubation behaviour, an important period of parental care in birds.

Incubation is an energetically demanding behaviour (Tinbergen and Williams 2002) and variation in incubation effort can have important consequences for fitness (Komdeur et al. 2002, Reid et al. 2002, Larsen et al. 2003, Gorman and Nager 2004). Male contribution to incubation is variable, but the factors affecting this variation are poorly understood (Reid et al. 2002). The most common pattern is incubation shared by both parents (Deeming 2002), and males can make a considerable contribution to the incubation of the offspring, giving females good reason to be choosy. Yet in other respects, incubation is a very different form of parental care in comparison to egg or chick provisioning. Whilst parents adjust their incubation effort in response to the temperature of the eggs (White and Kinney 1974), the division of incubation is largely a negotiation between two players and incubation effort is not divided between individual offspring but provided to the whole clutch. Moreover, whereas variation in female care to nestlings might be attributable to factors that are independent of the mate's current behaviour, incubation might be a more likely phase for parental negotiation (Schwagmeyer et al. 2002). This makes incubation particularly interesting for testing hypotheses concerning biparental care and provides an ideal opportunity to test theories of parental care allocation decisions.

Here, we aimed to experimentally test the prediction of the differential allocation hypothesis that a bird will put more effort into incubation when mated with an attractive mate in order to maximise its own reproductive potential. We therefore compared the incubation share of male and female zebra finches Taeniopygia guttata when attractiveness was manipulated using ring colour manipulation. Red rings have previously been found to make male zebra finches more attractive to and preferred by females in mate-choice trials, while green rings render them unattractive (Burley et al. 1982, Burley 1988b, Hunt et al. 1997). Failure to replicate this effect of ring colour on male attractiveness (Ratcliffe and Boag 1986) has been suggested to be due to inadequate lighting conditions (Hunt et al. 1997). A bird's behaviour towards its mate depends on the social environment (Magrath and Elgar 1997, Vignal et al. 2004). We therefore allowed the birds social interactions with conspecifics of the same and opposite sex by releasing them into outdoor aviaries to form small colonies. This is similar to Burley's experiments (1986, 1988a), and ensures that the behaviours observed are as representative of those in the wild as possible and the ring colour experiment is done under natural light. However, in Burley's tests both attractive and unattractive males were placed into the same colonies and thus, treatment-related assortative mating might have confounded interpretation of the results (Sheldon 2000). In order to prevent such assortative mating between treatments, all males within a colony were given the same ring colour. Female zebra finches are known to still differentially allocate parental effort when they cannot chose between two males of different attractiveness simultaneously (Swaddle 1996). However, males within a colony may also show natural variation in attractiveness, and attractive males have been found to pair with the earliest laying females (de Lope and Møller 1993, Badyaev and Hill 2002). Hence we included timing of laying in the analysis. We predict that a female paired with an attractive partner that is a red-ringed male and/or laying early, will put more effort into incubation than one mated to a less attractive male.

\section{Materials and methods}

We set up six colonies of captive-bred zebra finches on the Garscube Estate campus of the University of Glasgow, Glasgow, Scotland, UK, two in 2001 (AugustSeptember) and four in 2002 (June-July). Each colony was placed in a roofed outdoor aviary $(2.8 \times 5.5 \times$ $2.5 \mathrm{~m})$ with a sheltered indoor area $(0.9 \times 1.3 \times 1.0 \mathrm{~m}$, total volume of each aviary $\left.=39.7 \mathrm{~m}^{3}\right)$. Each colony consisted of 20 male and 21 female zebra finches at the start of the experiment and different birds were used in 2001 and 2002. The birds were fed a diet of ad libitum water and mixed seed (foreign finch food, supplied by Haith's, Cleethorpes, Lincolnshire, UK) containing pearl white, yellow, panicum, red, Japanese and Canadian canary millets. They also received greens (sprouted beans) and spray millet once per week, and a protein supplement consisting of egg-biscuit, poppy seeds (blue 
maw) and vitamins (Haith's, Cleethorpes) twice per week. Appropriate vitamin and calcium supplements for breeding birds (Daily Essentials 2 and Calcivet, produced by the Birdcare Company, Vetafarm Europe Ltd., Nailsworth, Gloucestershire, UK) were provided twice and five times per week, respectively. The birds received ad libitum oyster grit and cuttlebone.

\section{Experimental design}

Zebra finches were released into outdoor aviaries (two aviaries in 2001 and four aviaries in 2002) where all males were either ringed with a red or a green ring. All birds had previously bred with partners of unmanipulated attractiveness as individual pairs in small cages. To control for prior experience, birds were allocated to the aviaries with only unfamiliar birds of the opposite sex in the same aviary i.e., no previous mates or offspring and siblings of the opposite sex. Females all received a numbered red leg-ring for identification purposes (red is a neutral colour with respect to the attractiveness of female zebra finches (Burley et al. 1982)). The birds were weighed (to the nearest $0.1 \mathrm{~g}$ ) and tarsus length measured (to the nearest $0.05 \mathrm{~mm}$ ) on the day they were released into the aviaries (measurements for three males and three females are missing).

After release into the aviaries, the birds were left to acclimate for two weeks and then provided with nesting material and an excess of nestboxes. Each nest was checked daily for laying and we recorded latency to lay (the interval between opening the nestboxes and laying of the first egg) and clutch size. Some eggs were removed from the nest (for another part of the experiment) two days after clutch completion and replaced with an equal number of artificial eggs made of Fimo polymer clay (Eberhard Faber, Neumarkt, Germany). There was no difference in the incubation attentiveness between birds sitting on real or artificial eggs (mixed model (see statistical analysis below), male behaviour, egg type: $\mathrm{F}_{1,8.04}=0.29, \mathrm{P}=0.55$, colony: $\mathrm{Z}=0.63, \mathrm{P}=0.53$; female behaviour, egg type: $\mathrm{F}_{1,9.68}=0.49, \mathrm{P}=0.50$, colony: $\mathrm{Z}=$ $0.47, \mathrm{P}=0.64)$. Hence, birds incubated their natural clutch size but some pairs did not produce offspring.

Incubation was assumed to begin (day 1 of incubation) on the day on which the fourth egg was laid where the clutch size was five or more, and when the last egg was laid for clutches of less than five (Zann 1996). Incubation behaviour of a random selection of nests within each colony was recorded for a period of three hours using infra-red video cameras mounted above the nest. Observations on incubation behaviour of captive zebra finches are repeatable across the incubation period (females: $r=0.82 \pm 0.25$, males: $r=0.34 \pm 0.25$, $\mathrm{P}<0.02$, Gorman and Nager 2003). Observations from a total of 35 nests (20 with red-ringed males and 15 with green-ringed males) where at least one partner attended the clutch were used in the analysis. Three nests were observed twice and in these cases incubation behaviour was averaged over the multiple observation periods so there was one value per nest. As incubation behaviour may vary with time of day, day of incubation, clutch size and ambient conditions (Smith and Montgomerie 1992, Conway and Martin 2000) we controlled for these factors. We therefore recorded incubation in the early afternoon (average start time of the observation: 13.5 hours \pm 15.6 minutes, $\mathrm{N}=35$ ) during the middle third of the incubation period (between incubation days $5-8$, average age: $6.1 \pm 0.35$ days, $\mathrm{N}=35$ ). For each recording we noted the ambient temperature on site at the start of the observation and number of eggs incubated and included these as covariates in the statistical analysis.

The observers of the videotapes (H.E.G. and R.G.N.) were unaware of the treatment group of each of the observed nests. The times of the beginning and end of each incubation bout and the sex of each attending parent were recorded. The sex of the attending parent was readily identified due to sexual plumage dimorphism. Incubation attentiveness was defined as the total time for which the eggs were covered during each observation period and we calculated female incubation effort as:

\section{Female attentiveness/(female attentiveness \\ + male attentiveness).}

This measure was arcsine-transformed for the analysis. In the sample of the observed nests that incubated real eggs $(N=17)$, we recorded the number of eggs that successfully hatched and related this to the observed incubation behaviour.

\section{Statistical analyses}

We analysed variation in incubation behaviour in relation to male ring colour, ambient temperature, clutch size and latency. There were three replicate colonies within each ring colour treatment and birds within the same colony cannot be treated as independent data points. We have therefore included colony identity as a random effect into a generalised linear mixed model and used the Satterthwaite correction for degrees of freedom (Littell et al. 1996). Analyses were done in SAS (Version 8.2) using PROC MIXED. The random effect of colony identity (Z-statistics) was always controlled for, even when it was not statistically significant.

To analyse the data on the proportion of females laying in relation to treatment and the proportion of eggs that successfully hatched in relation to total incubation attentiveness of the male and female we used a mixed model with a binomial error distribution 
and a logit link function (macro GLIMMIX, SAS). All tests are two-tailed, while $\mathrm{P}<0.05$ is significant. Only significant interaction terms are reported. Means \pm standard errors are reported.

\section{Results}

At the beginning of the experiment, there were no differences between the treatment groups in body mass (mixed model, males: $\mathrm{F}_{1,3.86}=0.002, \mathrm{P}=0.97$; females: $\mathrm{F}_{1,3.18}=0.002, \quad \mathrm{P}=0.96$ ), or tarsus length (males: $\mathrm{F}_{1,4.03}=0.26, \mathrm{P}=0.63$; females: $\mathrm{F}_{1,4.02}=1.77, \mathrm{P}=0.25$ ). A similar proportion of females laid eggs in the two treatments $\left(\chi_{1}^{2}=0.62, \mathrm{P}=0.43\right)$, and overall $77.0 \%$ of females produced a clutch. Among the birds that were observed during incubation, the median latency to lay was 7 days after opening the nestboxes and did not differ from the unobserved birds (mixed model, $F_{1,93.8}=0.20$, $\mathrm{P}=0.66$ ), or between male ring colour treatments $\left(F_{1,3.32}=0.13, P=0.74\right)$. Clutch size also did not differ between nests where incubation behaviour was recorded and unobserved nest $\left(\mathrm{F}_{1,91.3}=1.37, \mathrm{P}=0.44\right)$ and was not influenced by male ring colour $\left(\mathrm{F}_{1,4.01}=0.13, \mathrm{P}=\right.$ 0.41 ), but tended to decrease with increasing latency to lay $\left(\mathrm{F}_{1,92.8}=3.59, \mathrm{P}=0.06\right)$.

Within a pair, on average females spent more time on a clutch than their partners $(\mathrm{N}=35$ pairs, females: $53.9 \pm 4.8 \%$, males: $36.4 \pm 4.1 \%$, paired t-test, $\mathrm{t}_{34}=2.04$, $\mathrm{P}=0.05)$. Female incubating a larger clutch incubated for a larger proportion of the total attentiveness time than females incubating a smaller clutch and female incubation effort declined with increasing ambient temperature (Table 1). The effect of male attractiveness on female incubation effort depended on the latency to lay (interaction between ring colour and latency: $\mathrm{P}=$ 0.04, see Table 1 and Fig. 1). Among earlier-laying pairs, females mated to a red-ringed males had a higher incubation effort than females with green-ringed males and their incubation effort declined with increasing latency (mixed model, $\mathrm{N}=20$, colony identity: $\mathrm{Z}=$ $2.47, \mathrm{P}=0.01$; latency: $\left.\mathrm{F}_{1,15}=13.55, \mathrm{P}=0.002\right)$. Incubation effort of females paired to red-ringed males declined

Table 1. Variation in female incubation effort (expressed as her proportional share of total attentiveness) in relation to male colour ring manipulation, latency to lay, ambient temperature and clutch size. Colony identity was entered as a random factor $(\mathrm{Z}=3.66, \mathrm{P}<0.001)$ in the mixed model (see Methods). Estimates are least squares means parameter estimates.

\begin{tabular}{llccr}
\hline Source of variation & df & F & P & \multicolumn{1}{c}{ Estimate } \\
\hline Constant & & & & $1.095 \pm 0.421$ \\
Ring colour & $1,28.2$ & 4.57 & 0.04 & $0.545 \pm 0.255$ \\
Latency & 1,29 & 0.47 & 0.50 & $0.019 \pm 0.024$ \\
Clutch size & $1,27.4$ & 6.92 & 0.01 & $0.113 \pm 0.043$ \\
Ambient temperature & 1,25 & 7.96 & 0.01 & $-0.064 \pm 0.023$ \\
Ring colour $\times$ latency & $1,26.6$ & 4.75 & 0.04 & $-0.059 \pm 0.027$
\end{tabular}

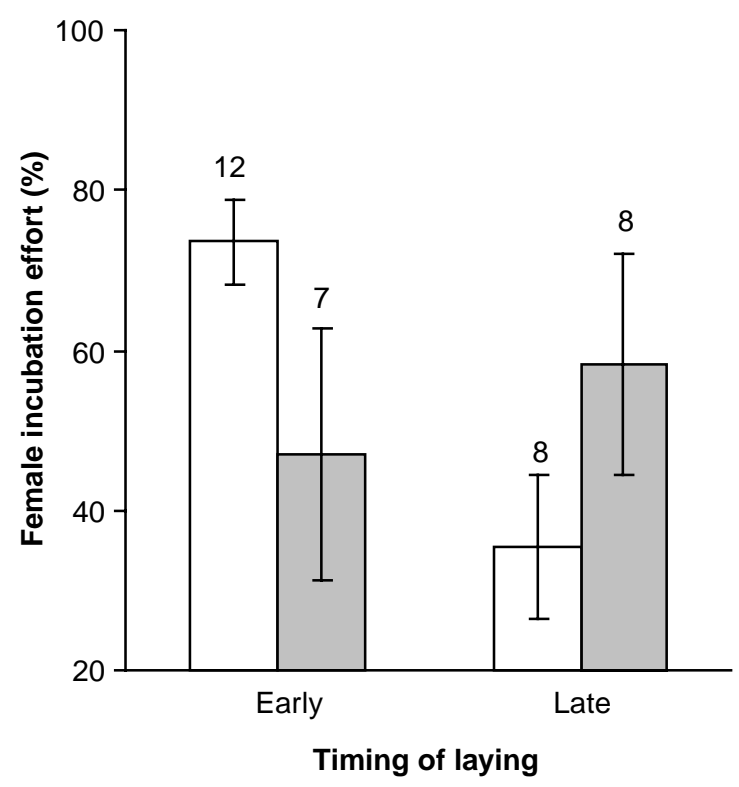

Fig. 1. Mean female incubation effort $( \pm S E)$ of zebra finches in relation to their partner's ring colour and the timing of laying. Males were either made more attractive by addition of red leg rings (open bars) or less attractive by addition of green rings (shaded bars). Female incubation effort is the proportion of the total attentiveness time that the females spent incubating. Pairs were grouped into early pairs (started laying before the average laying date) and late pairs (started laying after the average laying date) for clarity of graphical presentation only. Numbers above the bars refer to number of pairs in each group.

by $5.47 \pm 1.49 \%$ for each day the start of laying was delayed. No such difference was found among females paired to green-ringed males $(\mathrm{N}=15$, colony identity: $\mathrm{Z}=0.30, \mathrm{P}=0.76$; latency: $\left.\mathrm{F}_{1,9.15}=1.02, \mathrm{P}=0.34\right)$. This test had a power of 0.91 (Faul and Erdfelder 1992) to detect a similar decline in female incubation effort with latency as that found in females paired with red-ringed males.

Mean total attentiveness of males and females together was $90.2 \pm 2.6 \%(\mathrm{~N}=35)$. Total attentiveness of both parents together did not differ with latency to lay $\left(\mathrm{F}_{1,25.9}=0.20, \quad \mathrm{P}=0.66\right)$, or ring colour of males $\left(\mathrm{F}_{1,3.16}=0.48, \mathrm{P}=0.53\right)$. Reduced total attentiveness resulted in a significant decrease in hatching success $\left(\mathrm{N}=17, \chi_{1}^{2}=10.11, \mathrm{P}=0.001\right.$; male ring colour: $\chi_{1}^{2}=$ $0.81, \mathrm{P}=0.37)$.

\section{Discussion}

Our study found that, in zebra finches breeding in small colonies in outdoor aviaries, the relative share of female incubation behaviour was dependent on experimentally manipulated male attractiveness and timing of breeding. Among females mated to attractive males, early laying females had a higher relative share of incubation effort 
than late laying females. There was no difference in the incubation behaviour of females mated to unattractive mates in relation to latency to lay and females had a similar share in incubation behaviour to their partners. Overall nest attentiveness, however, was not significantly influenced by male attractiveness and timing of laying. These observations are only partly consistent with the predictions of the differential allocation hypothesis.

Our recordings of incubation behaviour consisted of one three-hour period during the middle of the incubation period. We believe this is a reliable measure of incubation effort of zebra finches. First, the main effect of this short observation period will be to add random error to our estimate of incubation effort and would cause a reduction in our ability to detect a difference. Therefore our test is conservative. Second, it is expected that incubation effort is related to hatching success (Nilsson and Smith 1988, Komdeur et al. 2002, Reid et al. 2002). We found the predicted positive relationship between our measure of incubation effort and hatching success, indicating that the short-term incubation effort is a reliable indicator of overall incubation effort in this species.

We observed an increase in female incubation effort with increasing clutch size and this could be explained by the differential allocation hypothesis. It has been suggested that clutch size in zebra finches is influenced by male attractiveness (Zann 1994, Balzer and Williams 1998) and the observed increase in effort of females incubating larger clutches could be seen as supporting the differential allocation hypothesis. However, in our study, we found no differences in clutch size between the treatments of male attractiveness.

The increased incubation effort of females mated to an attractive male may arise either because females put more effort into incubation to obtain and/or maintain an attractive mate or because attractive males reduce their share in incubation effort, but the total effort remains the same (Burley 1986). We will discuss both these possibilities in turn. First, females mated to red-ringed males may have been responding to the attractiveness of their mates and invested more into incubation. However, among red-ringed males, this effect was only found in early-laying pairs but not in late-laying pairs. This could be due to later-pairing males being perceived as less attractive as earlier-pairing males (de Lope and Møller 1993, Badyaev and Hill 2002). No such change in incubation effort with latency to lay was found in the treatment where male attractiveness was decreased. Female reproductive decisions are likely to be influenced by several male attributes (Collins et al. 1994). In our experiment, we manipulated ring colour only, but the males were likely to show natural variation in other characteristics of attractiveness such as, for example, song and/or bill colour. Hence, there is likely to be considerable variation in male attractiveness within the population that cannot be entirely overridden by the manipulation of male ring colour, and females may have responded to multiple signals in their allocation decision to incubation effort. Female zebra finches may only increase their incubation effort when paired with the very best males: those that appeared attractive on the basis of several traits. If this is the case, it would explain why a change in incubation effort with laying date was not seen in females mated to green-ringed males. Regardless of the timing of laying, green-ringed males may have had less variation in attractiveness and/or an overall attractiveness that was insufficient to stimulate greater female investment.

Secondly, the most attractive males may continue to pursue additional breeding opportunities and are trading-off future mating opportunities against parental effort into the current brood (Burley 1986, Qvarnström 1997, Magrath and Komdeur 2003, Qvarnström et al. 2003) or decrease parental effort (Sanz 2001). For example, male contribution to incubation in fairy martins Hirundo ariel decreased with increasing opportunities for extra-pair copulation (Magrath and Elgar 1997). In aviaries, male zebra finches pursue a moderate rate of extra-pair copulation (Birkhead et al. 1989). However, as the laying period progresses, fewer additional mating opportunities are available, and hence males mated to later-laying females had more time left to invest into incubation. There may thus be a price to pay for a female who has a very attractive mate, as he will do less, so she must invest more into incubation in order to maintain optimal conditions for embryo development. The absence of statistically significant differences in overall attentiveness with male attractiveness suggests that zebra finch females may have shown some compensation for the reduction in their partner's effort, at least our test could not detect a difference in total attentiveness between treatment groups. Although theoretical models predict that females should not raise their own effort enough to make up for the difference completely (Houston and Davies 1985, Winkler 1987, McNamara et al. 1999), differences in the extent of compensation are likely to depend on the costs of decreased parental effort (Sanz et al. 2000). In biparentally incubating birds one partner may only be able to reduce their contribution as long as the other partner can compensate and maintain incubation effort at a level where offspring can still successfully develop (Jones et al. 2002). In addition, in a captive colony such as that used in our study in which ad libitum food is provided, the costs of compensation may be small, allowing some compensation to occur. In future, to conclude whether the observed changes in male and female contribution to incubation represents evidence for increased effort in response to mate attractiveness or compensation for reduced effort of attractive males we would need to be able to determine which sex was controlling the division of incubation behaviour 
within the pair. We are at present unable to determine whether the male or the female drove the pattern of behaviour seen here. It would be of interest to compare the results of our study (and other investigations of the differential allocation hypothesis that have used a species with biparental care) with those of studies using a uniparental care, in which compensation would not be an issue.

An alternative interpretation of the observed pattern of incubation effort could be that there is also variation in female quality within a population. Females of higher quality could have been mating assortatively with the more attractive males. Since we only used one ring colour per aviary, we can exclude such treatment-related assortative pairing. However, there might be some assortative pairing occurring within aviaries. Females that lay early have been suggested to be those in the best condition (Price et al. 1988, Moreno et al. 1998), and hence may be more attractive to males (Wynn and Price 2002). Late-laying females may have been unable or unwilling to increase their incubation effort in response to male attractiveness, because they might not be able to afford the costs of increased incubation effort. This would still be in agreement with the differential allocation hypothesis, as it predicts that only individuals likely to capitalise on increased parental effort should increase parental effort in relation to male attractiveness (Sheldon 2000). However, often the costs and benefits of altered reproductive decisions in relation to male attractiveness are not known. In zebra finches, we have shown that an increase in incubation effort improves hatching success (this study) and offspring quality (Gorman and Nager 2004). On the other hand, increased incubation effort can have negative effects on later reproductive performance (Heaney and Monaghan 1996, Reid et al. 2000), but such data on the effects of incubation effort on later stages of reproduction are lacking for zebra finches. The higher incubation effort of early-laying females paired to attractive males could also be due to the female's better quality or condition compared to late-laying females, rather than male attractiveness (Rintamäki et al. 1998). We would then expect the same relationship between latency and incubation effort in females paired to green-ringed males, as there would have been a similar within-population variation in female quality. However, we found no such relationship in this treatment group, supporting the interpretation that the observed pattern was due to differences in mate attractiveness rather than differences in female quality.

In conclusion, we found evidence that the division of labour during incubation between the sexes varied with male attractiveness and timing of breeding. We cannot distinguish between the two mechanisms that could cause this pattern. Either females in good condition and/or paired to more attractive males increased their incubation effort, or males with greater potential to attract additional breeding partners decreased their investment, causing females to compensate. The two explanations are not mutually exclusive and can occur simultaneously; an attractive male could withhold a proportion of parental care that is smaller than the extra care invested by the female, thus leading to an overall higher level of care despite the male's comparatively lower input (Burley 1988a, Møller and de Lope 1995). Our experiment suggests that it is important to consider that, in a more realistic situation such as the social environment in which breeding takes place a variety of stimuli may contribute to parental investment decisions. In studies with caged individual pairs, the range of decisions available to each member of the pair are reduced, leading to results that may not be representative of natural behaviour. Additionally, our results stress the importance of considering the division of parental duties between both parents, and not only the response of one parent, when studying parental care decisions in wild populations.

Acknowledgements - We thank Graham Law, Paul McGowan, Davina Graham, John Laurie and Alistar Kirk for caring for the birds; Kat Fellerman for her help, the Electronic Workshop for assistance with the video equipment and two anonymous reviewers for their constructive comments that greatly improved the presentation. H.E.G. was funded by a studentship from the Natural Environment Research Council. K.E.A. is funded by a Royal Society University Research Fellowship.

\section{References}

Andersson, M. 1986. Evolution of condition-dependent sex ornaments and mating preferences: sexual selection based on viability differences. - Evolution 40: 804-816.

Badyaev, A. V. and Hill, G. E. 2002. Paternal care as a conditional strategy: distinct reproductive tactics associated with elaboration of plumage ornamentation in the house finch. - Behav. Ecol. 13: 591-597.

Balzer, A. L. and Williams, T. D. 1998. Do female zebra finches vary primary reproductive effort in relation to mate attractiveness? - Behaviour 135: 297-309.

Birkhead, T. R., Hunter, F. M. and Pellat, J. E. 1989. Sperm competition in the zebra finch Taeniopygia guttata. - Anim. Behav. 38: 935-950.

Burley, N. T., Krantzberg, G. and Radman, P. 1982. Influence of colour-banding on the conspecific preferences of zebra finches. - Anim. Behav. 30: 444-455.

Burley, N. T. 1986. Sexual selection for aesthetic trait in species with biparental care. - Am. Nat. 127: 415-445.

Burley, N. T. 1988a. The differential-allocation hypothesis: an experimental test. - Am. Nat. 132: 611-628.

Burley, N. T. 1988b. Wild zebra finches have band-colour preferences. - Anim. Behav. 36: 1235-1237.

Clutton-Brock, T. H. 1991. The evolution of parental care. - University Press, Princeton.

Collins, S. A., Hubbard, C. and Houtman, A. M. 1994. Female mate choice in the zebra finch-the effect of male beak colour and male song. - Behav. Ecol. Sociobiol. 35: 21-25.

Conway, C. J. and Martin, T. E. 2000. Effects of ambient temperature on avian incubation behaviour. - Behav. Ecol. 11: $178-188$.

Cunningham, E. J. A. and Russell, A. F. 2000. Egg investment is influenced by male attractiveness in the mallard. - Nature 404: $74-77$. 
de Lope, F. and Møller, A. P. 1993. Female reproductive effort depends on the degree of ornamentation of their mates. - Evolution 47: 1152-1160.

Deeming, D. C. 2002. Avian incubation. - Oxford University Press, Oxford.

Drickamer, L. C., Gowaty, P. A. and Holmes, C. M. 2000. Free female mate choice in house mice affects reproductive success and offspring viability and performance. - Anim. Behav. 59: 371-378.

Faul, F. and Erdfelder, E. 1992. GPOWER: A priori, post-hoc, and compromise power analysis for MS-DOS. - Dept. of Psychology, Bonn University, Bonn, Germany.

Gil, D., Graves, J., Hazon, N. and Wells, A. 1999. Male attractiveness and differential testosterone investment in zebra finch eggs. - Science 286: 126-128.

Gorman, H. E. and Nager, R. G. 2003. State-dependent incubation behaviour in the zebra finch. - Anim. Behav. 65: $745-754$.

Gorman, H. E. and Nager, R. G. 2004. Prenatal developmental conditions have long-term effects on offspring fecundity. - Proc. R. Soc. B 271: 1923-1928.

Hamilton, W. D. and Zuk, M. 1982. Heritable true fitness and bright birds: a role for parasites? - Science 218: 384-387.

Heaney, V. and Monaghan, P. 1996. Optimal allocation of effort between reproductive phases: the trade-off between incubation costs and subsequent brood rearing capacity. - Proc. R. Soc. B 263: 719-1724.

Hoelzer, G. 1989. The good parent process of sexual selection. - Anim. Behav. 38: 1067-1078.

Houston, A. I. and Davies, N. B. 1985. The evolution of co-operation and life history in the dunnock, Prunella modularis. - In: Sibly, R. M. and Smith, R. H. (eds). Behavioural ecology: ecological consequences of adaptive behaviour. Blackwell, Oxford, pp. 471-487.

Hunt, S., Cuthill, I. C., Swaddle, J. P. and Bennett, A. T. D. 1997. Ultraviolet vision and band-colour preferences in female zebra finches, Taeniopygia guttata. - Anim. Behav. 54: $1383-1392$.

Jennions, M. D. and Petrie, M. 2000. Why do females mate multiply? A review of the genetic benefits. - Biol. Rev. 75: $1-64$.

Jones, K. M. G., Ruxton, G. D. and Monaghan, P. 2002. Model parents: is full compensation for reduced partner nest attendance compatible with stable biparental care? - Behav. Ecol. 13: 838-843.

Keyser, A. and Hill, G. E. 2000. Structurally-based plumage colour is an honest indicator of quality in male blue grosbeaks. - Behav. Ecol. 11: 202-209.

Kolm, N. 2001. Females produce larger eggs for large males in a paternal mouthbrooding fish. - Proc. R. Soc. B 268: 22292234.

Komdeur, J., Wiersma, P. and Magrath, M. 2002. Paternal care and male mate-attraction effort in the European starling is adjusted to clutch size. - Proc. R. Soc. B 269: 1253-1261.

Larsen, V. A., Lislevand, T. and Byrkjedal, I. 2003. Is clutch size limited by incubation ability in northern lapwings? - J. Anim. Ecol. 72: 784-792.

Littell, R. C., Milliken, G. A., Stroup, W. W. and Wolfinger, R. D. 1996. SAS system for mixed models. - SAS Institute Inc., Cary, NC, USA.

Magrath, M. J. L. and Elgar, M. 1997. Paternal care declines with increased opportunity for extra-pair mating in fairy martins. - Proc. R. Soc. B 264: 1731-1736.

Magrath, M. J. L. and Komdeur, J. 2003. Is male care compromised by additional mating opportunity? - Trends. Ecol. Evol. 18: 424-430.

McNamara, J. M., Gasson, C. E. and Houston, A. I. 1999. Incorporating rules for responding into evolutionary games. - Nature 401: 368-371.

Moreno, J., de Leon, A., Fargallo, J. A. and Moreno, A. 1998. Breeding time, health and immune response in the chinstrap penguin Pygoscelis antarctica. - Oecologia 115: $312-$ 319 .
Møller, A. P. and de Lope, F. 1995. Differential allocation and sexual ornamentation. - Evolution 49: 1290-1292.

Nilsson, J. A. and Smith, H. G. 1988. Incubation feeding as a male tactic for early hatching. - Anim. Behav. 36: 641-647.

Norris, K. 1990. Female choice and quality of parental care in the great tit Parus major. - Behav. Ecol. Sociobiol. 27: 275281.

Petrie, M. and Williams, A. 1993. Peahens lay more eggs for peacocks with larger trains. - Proc. R. Soc. B 251: 127-131.

Price, T., Kirkpatrick, M. and Arnold, S. J. 1988. Directional selection and the evolution of breeding date in birds. - Science 240: 798-799.

Qvarnström, A. 1997. Experimentally increased badge size increases male competition and reduces male parental care in the collared flycatcher. - Proc. R. Soc. B 264: 1225-1231.

Qvarnström, A., Sheldon, B. C., Pärt, T. and Gustafsson, L. 2003. Male ornamentation, timing of breeding, and cost of polygyny in the collared flycatcher. - Behav. Ecol. 14: $68-73$.

Ratcliffe, L. M. and Boag, P. T. 1986. Effects of colour bands on male competition and sexual attractiveness in zebra finches (Poephilia guttata). - Can. J. Zool. 65: 333-338.

Reid, J. M., Monaghan, P. and Ruxton, G. D. 2000. Resource allocation between reproductive phases: the importance of thermal conditions in determining the cost of reproduction. - Proc. R. Soc. B 267: 37-41.

Reid, J. M., Monaghan, P. and Ruxton, G. D. 2002. Males matter: the occurrence and consequences of male incubation in starlings (Sturnus vulgaris). - Behav. Ecol. Sociobiol. 51: 255-261.

Reyer, H-U., Frei, G. and Som, C. 1999. Cryptic female choice: frogs reduce clutch size when amplexed by undesired males. - Proc. R. Soc. B 266: 2101-2107.

Rintamäki, P. T., Lundberg, A., Alatalo, R. V. and Höglund, J. 1998. Assortative mating and female clutch investment in black grouse. - Anim. Behav. 56: 1399-1403.

Rohde, P. A., Johnsen, A. and Lifjeld, J. T. 1999. Parental care and sexual selection in the bluethroat, Luscinia s. svecica: a field-experimental test of the differential allocation hypothesis. - Ethology 105: 651-663.

Saino, N., Bertacche, V., Ferrari, R. P., Martinelli, R., Møller, A. P. and Stradi, R. 2002a. Carotenoid concentration in barn swallow eggs is influenced by laying order, maternal infection and paternal ornamentation. - Proc. R. Soc. B 269: 1729-1733.

Saino, N., Ferrari, R. P., Martinelli, R., Romano, M., Robulini, D. and Møller, A. P. 2002b. Early maternal effects mediated by immunity depend on sexual ornamentation of the male partner. - Proc. R. Soc. B 269: 1005-1009.

Sanz, J. J. 2001. Experimentally reduced male attractiveness increases parental care in the pied flycatcher Ficedula hypoleuca. - Behav. Ecol. 12: 171-176.

Sanz, J. J., Kranenbarg, S. and Tinbergen, J. M. 2000. Differential response by males and females to manipulation of partner contribution in the great tit (Parus major). - J. Anim. Ecol. 69: 74-84.

Schwagmeyer, P. L., Mock, D. W. and Parker, G. A. 2002. Biparental care in house sparrows: negotiation or sealed bid? - Behav. Ecol. 13: 713-721.

Sheldon, B. C. 2000. Differential allocation: tests, mechanisms and implications. - Trends. Ecol. Evol. 15: 397-402.

Siefferman, L. and Hill, G. E. 2003. Structural and melanin coloration indicates parental effort and reproductive success in male eastern bluebirds. - Behav. Ecol. 14: 855-861.

Simmons, L. W. 1987. Female choice contributes to offspring fitness in the field cricket, Gryllus bimaculatus (De Geer). - Behav. Ecol. Sociobiol. 21: 313-321.

Smiseth, P. T., Örnborg, J., Andersson, S. and Amundsen, T. 2001. Is male plumage reflectance correlated with paternal care in bluethroats? - Behav. Ecol. 12: 164-170.

Smith, H. G. and Montgomerie, R. 1992. Male incubation in barn swallows: the influence of nest temperature and sexual selection. - Condor 94: 750-759. 
Swaddle, J. P. 1996. Reproductive success and symmetry in zebra finches. - Anim. Behav. 51: 203-210.

Swaddle, J. P. and Cuthill, I. C. 1994. Preference for symmetric males by female zebra finches. - Nature 367: 165-166.

Tinbergen, J. M. and Williams, J. B. 2002. Energetics of incubation. - In: Deeming, D. C. (ed.). Avian incubation. Oxford University Press, Oxford, pp. 299-313.

Torres, R. and Velando, A. 2003. A dynamic trait affects continuous pair assessment in the blue-footed booby, Sula nebouxii. - Behav. Ecol. Sociobiol. 55: 65-72.

Vignal, C., Mathevon, N. and Mottin, S. 2004. Audience drives male songbird response to partner's voice. - Nature 430: $448-451$.

White, F. N. and Kinney, J. L. 1974. Avian incubation. - Science 186: 107-115.
Winkler, D. W. 1987. A general model for parental care. - Am. Nat. 130: 526-543.

Wynn, S. E. and Price, T. 2002. Male and female choice in zebra finches. - Auk 110: 635-638.

Zahavi, A. 1975. Mate selection-a selection for a handicap. - J. Theor. Biol. 53: 205-214.

Zann, R. A. 1994. Effects of band colour on survivorship, body condition and reproductive effort of free-living Australian zebra finches. - Auk 111: 131-142.

Zann, R. A. 1996. The zebra finch: a synthesis of field and laboratory studies. - Oxford University Press, Oxford.

(Received 18 May 2004, revised 17 September 2004, accepted 22 September 2004.) 